УДК 519.21

\title{
On the Limit Distribution of Sums of Real Random Variables
}

Sergey V. Chebotarev*

Altai state pedagogical university

Molodezhnaya, 55, Barnaul, 656015

Russia

Received 28.10.2016, received in revised form 12.12.2016, accepted 10.03.2017

Considers centered sequence of absolutely continuous random variables with a non-trivial weak limit of the sums $\frac{1}{\sqrt{n}} \sum_{i=1}^{n} \xi_{i}$. We found a general view of the limit distribution. It is shown that the form of the limit distribution depends only on the average mixed moments of the first order, describing the sequence of Rademacher random variables, into which can be decomposed the elements of the given sequence.

Keywords: dependent random variables, the sums of dependent random variables, limit distribution of sums of random variables, the normality of the limit distribution of sums of random variables.

DOI: 10.17516/1997-1397-2017-10-3-310-313.

\section{Introduction}

In this paper we continue the study initiated in [1]. The main purpose is to obtain a general form of the limit distribution sums of centered absolutely continuous random variables with a non-trivial weak limit of the sums $\frac{1}{\sqrt{n}} \sum_{i=1}^{n} \xi_{i}$.

\section{Preliminary results}

Let us consider a sequence of absolutely continuous centered real random variables $\xi=$ $\left(\xi_{t}\right)_{t \in N}$. We assume that the random variables are defined at similar spaces of elementary events $\Omega_{t}=\Omega, t \in I$ with similar $\sigma$-algebras of events $\mathfrak{A}_{t}=\mathfrak{A}$. As previously, we assume $\Omega_{I}=\Omega_{1} \times \Omega_{2} \times \ldots \times \Omega_{t} \times \ldots$ and $\mathfrak{A}_{I}=\mathfrak{A}_{1} \times \mathfrak{A}_{2} \times \ldots \times \mathfrak{A}_{t} \times \ldots$ The space of values of random variables, we assume the set of real numbers $\xi_{t}(\omega) \in \mathfrak{X}_{t}=\mathbf{R}, \omega \in \Omega_{t}$ with given on it Borel $\sigma$-algebra $\mathfrak{B}$. By analogy with previous unite into the set $\Xi_{3}$ such the sequence of random variables $\xi=\left(\xi_{t}\right)_{t \in N}$, for which exists a continuous random variable $\eta_{\xi}$ - weak limit of sequence $S_{1 / 2}\left(\xi_{(n)}\right)=\frac{1}{\sqrt{n}} \sum_{t=1}^{n} \xi_{t}$

$$
\eta_{\xi} \stackrel{\text { weak }}{=} \lim _{n \rightarrow \infty} S_{1 / 2}\left(\xi_{(n)}\right) .
$$

We also denote $\hat{\Xi}_{3} \subset \Xi_{3}$ subset of sequences with averaged links (shortly sal ) $\hat{\xi}=\left(\hat{\xi}_{t}\right)_{t \in N}$, the existence and construction of which is shown in Theorem 3.3 [2] and for distribution functions of this sequences the following property satisfied:

$$
\mathbf{F}_{\eta_{\xi}}(y)=\mathbf{F}_{\eta_{\hat{\xi}}}(y), \quad \forall y \in \mathbf{R} .
$$

*svcheb@yandex.ru

(c) Siberian Federal University. All rights reserved 
We study the limit distribution of the random variable $\eta_{\xi}$ :

$$
\eta_{\xi} \stackrel{\text { weak }}{=} \lim _{n \rightarrow \infty} \frac{1}{\sqrt{n}} \sum_{t=1}^{n} \xi_{t}, \text { where } \xi=\left(\xi_{t}\right)_{t \in N} \in \Xi_{3} .
$$

In fact, to achieve this goal we needed to prove the existence of such a sequence $\hat{\gamma}=\hat{\gamma}(\xi) \in \hat{\Xi}_{1}$, that

$$
\mathbf{F}_{\eta_{\xi}}(x)=\mathbf{F}_{\eta_{\hat{\gamma}}}(x), \forall x \in \mathbf{R}
$$

and apply Theorem $3[1]$ to it. Issues of existence and the construction of sequences of this type for a given distribution of sums of elements of the initial sequences are described in [2]. Therefore, we will be based on results of this work.

\section{Main results}

Consider the approximation sums $S_{1 / 2}\left(\xi_{(n)}\right)=\frac{1}{\sqrt{n}} \sum_{t=1}^{n} \xi_{t}$ of random variables the investigated sequence $\xi$ by sums $S_{1 / 2}\left(\pi_{(s, n)}\right)=\frac{1}{\sqrt{n}} \sum_{t=1}^{n} \pi_{t, s, n}$ of lattice random variables $\pi_{t, s, n}$. For this purpose we divide the set of real numbers $\mathbf{R}$ as follows:

$$
\begin{aligned}
& \Delta x_{s}(k)=\left(\frac{2 k-s-1}{\sqrt{s}}, \frac{2 k-s+1}{\sqrt{s}}\right] \\
& \quad \text { for } k=1, \ldots, s-1, \Delta x_{s}(0)=\left(-\infty,-\frac{s-1}{\sqrt{s}}\right], \Delta x_{s}(s)=\left(\frac{s-1}{\sqrt{s}}, \infty\right) .
\end{aligned}
$$

At that we put

$$
\mathbf{P}\left(S_{1 / 2}\left(\pi_{(s, n)}\right)=\frac{2 k-s}{\sqrt{s}}\right)=\mathbf{P}\left(S_{1 / 2}\left(\xi_{(n)}\right) \in \Delta x_{n}(k)\right)=\mathbf{P}_{S_{1 / 2}}\left(\Delta x_{s}(k)\right), k=0,1, \ldots, s .
$$

For $\pi_{(s, n)}$ in [2] shown ( see Theorem 2.4) the existence of a finite $s a l \hat{\pi}_{(s, n)}$, having the same distribution of sums. Out there it also shown the existence of a finite and Rademacher type sal $\hat{\gamma}_{(s n)}$ such that

$$
\hat{\pi}_{t, s, n}=\frac{1}{\sqrt{s}} \sum_{i=0}^{s-1} \hat{\gamma}_{t+i \cdot n}
$$

For it also is performed the relation:

$$
\mathbf{F}_{S_{1 / 2}(\pi(n))}(x)=\mathbf{F}_{S_{1 / 2}\left(\hat{\gamma}_{(s n)}\right)} \forall x \in \mathbf{R}, \text { where } S_{1 / 2}\left(\hat{\gamma}_{(s n)}\right)=\frac{1}{\sqrt{s n}} \sum_{t=1}^{s n} \hat{\gamma}_{t} .
$$

Proceeding to the limit with $s \rightarrow \infty$, obtain the $\operatorname{sal} \hat{\gamma}_{(n, N)}$ for which

$$
\mathbf{F}_{S_{1 / 2}\left(\xi_{(n)}\right)}(x)=\mathbf{F}_{S_{1 / 2}\left(\hat{\gamma}_{(n N)}\right)} \forall x \in \mathbf{R}, \text { where } S_{1 / 2}\left(\hat{\gamma}_{(n N)}\right) \stackrel{\text { weak }}{=} \lim _{s \rightarrow \infty} \frac{1}{\sqrt{s n}} \sum_{t=1}^{s n} \hat{\gamma}_{t} \text {. }
$$

Note that $\hat{\gamma}_{(n, N)} \in \hat{\Xi}_{1}$. Further, proceeding to the limit with $n \rightarrow \infty$, obtain, on the one parties, sal $\hat{\gamma}$ for which

$$
\mathbf{F}_{\eta_{\xi}}(x)=\mathbf{F}_{\eta_{\hat{\gamma}}}(x) \forall x \in \mathbf{R}, \text { where } \eta_{\hat{\gamma}} \stackrel{\text { weak }}{=} \lim _{r \rightarrow \infty} \frac{1}{\sqrt{r}} \sum_{t=1}^{r} \hat{\gamma}_{t} .
$$


But on the other parties, random variables of $\boldsymbol{s a l} \hat{\xi}$, constructed by the limit sum of the original sequence $\xi$, nothing else than

$$
\hat{\xi}_{t} \stackrel{\text { weak }}{=} \lim _{n \rightarrow \infty} \lim _{s \rightarrow \infty} \hat{\pi}_{t, s, n} \text { or a considering (1), we have } \hat{\xi}_{t} \stackrel{\text { weak }}{=} \lim _{n \rightarrow \infty} \lim _{s \rightarrow \infty} \frac{1}{\sqrt{s}} \sum_{i=0}^{s-1} \hat{\gamma}_{t+i \cdot n} \text {. }
$$

Then

Lemma 1. Let us assume that a sequence $\xi \in \Xi_{3}$ is given. Then there exists a sequence $\hat{\gamma}=$ $\hat{\gamma}(\xi) \in \hat{\Xi}_{1}$ such that

$$
\mathbf{F}_{\eta_{\xi}}(x)=\mathbf{F}_{\eta_{\hat{\gamma}}}(x), \forall x \in \mathbf{R}
$$

Proof. Follows from the foregoing.

Theorem 1. Let us assume that a sequence $\xi \in \Xi_{3}$ is given. Then random variable $\eta$

$$
\eta \stackrel{\text { weak }}{=} \lim _{n \rightarrow \infty} \frac{1}{\sqrt{n}} \sum_{t=1}^{n} \xi_{t}
$$

have a density distribution function $\mu$ as follows:

$$
\mu(x)=\frac{1}{\sqrt{2 \pi}} e^{-\frac{x^{2}}{2}} \sum_{m=0}^{\infty} \ddot{v}_{m}(\hat{\gamma}) \cdot h_{m}(x), \forall x \in \mathbf{R}
$$

where $\ddot{v}_{m}$ are mixed moments of the sequence $\hat{\gamma}(\xi) \in \hat{\Xi}_{1}$, constructed in Lemma 1 .

Proof. Follows from Theorem 3 [1] and Lemma 1.

Theorem 2. Let us assume that a sequence $\xi \in \Xi_{3}$ is given. Then, if the density distribution function $\mu$ of the random variable $\eta$

$$
\eta \stackrel{\text { weak }}{=} \lim _{n \rightarrow \infty} \frac{1}{\sqrt{n}} \sum_{t=1}^{n} \xi_{t},
$$

is continuous function, all moments for this random variable are exist and finite.

Proof. Similarly to the proof Corollary $1[1]$.

Corollary 1. Let us assume that a sequence $\xi \in \Xi_{3}$ is given. Then a random variable $\eta$

$$
\eta \stackrel{\text { weak }}{=} \lim _{n \rightarrow \infty} \frac{1}{\sqrt{n}} \sum_{t=1}^{n} \xi_{t}
$$

have a standard normal distribution then and only then when

$$
\lim _{n \rightarrow \infty} \ddot{v}_{m}\left(\hat{\gamma}_{(n)}\right)=0, \forall m \geqslant 2 .
$$

Proof. Obviously follows from the expression for density of limit distribution in Theorem 1.

Concerning the moments of sequence of random variables $\hat{\xi}$ can be argued, considering relation (16) [1] and method of forming sequence $\hat{\gamma}$, that 
- if

$$
\ddot{v}_{m}(\hat{\xi})=\lim _{n \rightarrow \infty} \lim _{s \rightarrow \infty} s^{m} \cdot \ddot{v}_{m}\left(\hat{\gamma}_{(n), s}\right)=0, \forall m \geqslant 2,
$$

then the sequence $\hat{\xi}$ has zero average mixed moments for $m=2,3 \ldots$, that is particularly true for independent random variables;

- if

$$
\ddot{v}_{m}(\hat{\xi})=\lim _{n \rightarrow \infty} \lim _{s \rightarrow \infty} s^{m} \cdot \ddot{v}_{m}\left(\hat{\gamma}_{(n), s}\right)<\infty, \forall m \geqslant 2,
$$

then the sequence $\hat{\xi}$ has finite moments;

- and if beginning with some $m=m_{0}$

$$
\ddot{v}_{m}(\hat{\xi})=\lim _{n \rightarrow \infty} \lim _{s \rightarrow \infty} s^{m} \cdot \ddot{v}_{m}\left(\hat{\gamma}_{(n), s}\right)=\infty
$$

then moments $\left(m \geqslant m_{0}\right)$ of sequence $\hat{\xi}$ does not exist.

\section{References}

[1] S.V.Chebotarev, On Limit Distribution of Sums of Random Variables, Journal of Siberian Federal University. Mathematics \& Physics, 9(2016), no 1, 17-29.

[2] S.V.Chebotarev, About sequences of random variables with averaged links, Vestnik AltSPA, seriya: estestv. i tochnye nauki, $\mathbf{7}(2011), 28-37$ (in Russian).

\section{О предельном распределении сумм действительных случайных величин}

\section{Сергей В. Чеботарев}

Алтайский государственный педагогический университет Молодежная, 55, Барнаул, 656015

Россия

Рассмотрены центрированные последовательности абсолютно непрерывных случайных величин, имеющие нетривиалъный слабый предел сум⿻ $\frac{1}{\sqrt{n}} \sum_{i=1}^{n} \xi_{i}$. Для них найден общий вид предельного распределения. Показано, что вид предельного распределения зависит лишь от усредненных смешанных моментов первого порядка, характеризующих случайные величины последовательности радемахеровских случайных величин, в которую можно разложить элементы рассматриваемой последовательности.

Ключевые слова: зависимые случайные величины, сумма зависимых случайных величин, предельное распределение сумм случайных величин, нормальность предельного распределения сумм случайных величин. 